\title{
Anisotropic Thermal Expansion of Tetragonal Zirconia Polycrystals
}

\author{
Tzer-Shin Sheu ${ }^{\star, \dagger}$
}

Department of Materials Science and Engineering, The University of Michigan, Ann Arbor, Michigan $48109-2136$

Thermal expansion coefficients ( $\alpha_{a}$ and $\alpha_{c}$ ) in two crystallographic axes ( $a$ and $c$ ) of the tetragonal phase are measured at $25^{\circ}-1200^{\circ} \mathrm{C}$ in $\mathrm{ZrO}_{2}-\mathrm{M}_{2} \mathrm{O}_{3}(\mathrm{M}=\mathrm{Sc}$, In, Yb) and in $\mathrm{ZrO}_{2}-\mathrm{YTaO}_{4}$. The difference between these two thermal expansion coefficients, $\alpha_{c}-\alpha_{a}$, decreases with $\mathrm{M}_{2} \mathrm{O}_{3}$ or $\mathrm{YTaO}_{4}$ composition even though the tetragonality $(c / a)$ behaves differently in these two systems. The locus of $\alpha_{c}=$ $\alpha_{a}$ represents a maximum tetragonality for the tetragonal phase, but not the phase boundary for the cubic phase. The relationships among thermal expansion, temperature, and composition are discussed.

\section{Introduction}

D VALENT and trivalent metal oxides such as $\mathrm{MgO}, \mathrm{CaO}$, $\mathrm{Y}_{2} \mathrm{O}_{3}$, and rare-earth oxides have been found to be the stabilizing agents in zirconia solid solutions. ${ }^{1.2}$ This is because the tetragonal phase $(t)$ decreases both its tetragonality $(c / a)$ and its transformation temperature to the monoclinic phase $(m)$ by adding these oxides to the pure zirconia. ${ }^{36}$ Conversely, pentavalent metal oxides such as $\mathrm{Ta}_{2} \mathrm{O}_{5}$ and $\mathrm{Nb}_{2} \mathrm{O}_{5}$ act as destabilizers, ${ }^{6.7}$ because these oxides increase both the tetragonality and the transformation temperature of $t \rightarrow m$ when they are added to the 2 or 3 mol $\% \mathrm{Y}_{2} \mathrm{O}_{3}$-tetragonal zirconia polycrystalline material (TZP). Therefore, it is generally believed that the transformation temperature of $t \rightarrow m$ is correlated with tetragonality.

However, the above correlation does not exist in the $\mathrm{ZrO}_{2}-$ $\mathrm{YTaO}_{4}$ system, whose isothermal ternary oxide phase diagram at $1500^{\circ} \mathrm{C}$ is shown in Fig. 1. A stable tetragonal phase with a tetragonality larger than that in the pure zirconia has been reported to exist in the composition range 15-25 mol\% $\mathrm{YTaO}_{4}$ at $25^{\circ} \mathrm{C}{ }^{7}$ With composition less than $14.0 \mathrm{~mol} \% \mathrm{YTaO}_{4}, t \rightarrow m$ transformation temperature decreases with increasing $\mathrm{YTaO}_{4}$ composition. This suppression of $t \rightarrow m$ transformation temperature indicates that $\mathrm{YTaO}_{4}$ oxide is a stabilizing agent. However, there is no strong evidence to prove whether the unquenchable tetragonal phase with $0-14 \mathrm{~mol}_{\%} \mathrm{YTaO}_{4}$ has the same crystal structure as the stable tetragonal phase with 15-25 mol\% $\mathrm{YTaO}_{4}$. In this study, a lattice parameter measurement at high temperature is used to investigate the phase continuity in the composition range $0-25 \% \mathrm{YTaO}_{4}$.

Anisotropic thermal expansion behavior $\left(\alpha_{i} \neq \alpha_{i i}\right)$ has been reported to affect tetragonality $(c / a)$ for the tetragonal zirconia crystals, ${ }^{8-I 0}$ where $\alpha_{a}$ and $\alpha_{c}$ are thermal expansion coefficients for the crystallographic $a$ and $c$ axes, respectively. In the $\mathrm{ZrO}_{2}-$ $\mathrm{Y}_{2} \mathrm{O}_{3}$ system, $c / a$ and $\alpha_{i} / \alpha_{a}$ are temperature-dependent, and both of them decrease as $\mathrm{Y}_{2} \mathrm{O}_{3}$ composition increases at temperatures of $25^{\circ}-800^{\circ} \mathrm{C} .{ }^{8}$ In the pure zirconia, $c / a$ first increases and then decreases as temperature increases from $\sim 1100^{\circ} \mathrm{C}$ up to $\sim 2370^{\circ} \mathrm{C}$. ${ }^{9}$ This reverse $\mathrm{c} / \mathrm{a}$ behavior also exists in the $t^{\prime}$

D. B. Marshall-contributing editor

\footnotetext{
Manuscript No. 195164. Received March 26, 1992; approved March 29, 1993.

"Member, American Ceramic Society.

${ }^{4}$ Current address: Department of Biologic and Materials Science, School of Dentistry, University of Michigan, Ann Arbor, MI.
}

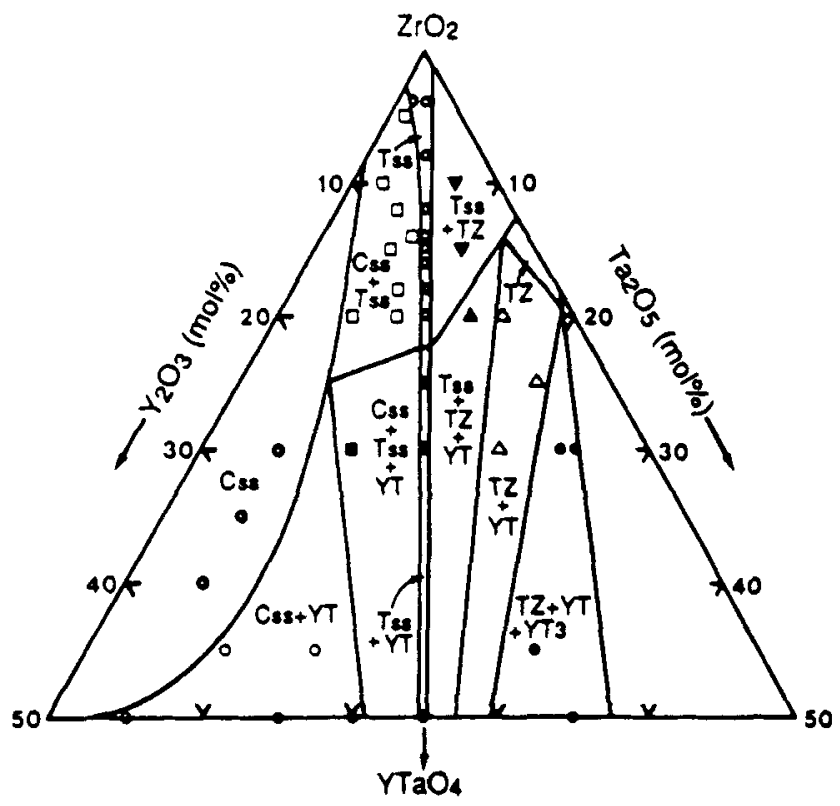

Fig. 1. Part of the ternary phase diagram for the system $\mathrm{ZrO}_{2}-\mathrm{Y}_{2} \mathrm{O}_{3}-$ $\mathrm{Ta}_{2} \mathrm{O}_{5}$ at $1500^{\circ} \mathrm{C}$. Tss is $t-\mathrm{ZrO}_{2}$ solid solution, $\mathrm{Css}$ is $c-\mathrm{ZrO}_{2}$ solid solution, $\mathrm{TZ}$ is $\mathrm{Ta}_{2} \mathrm{Zr}_{6} \mathrm{O}_{47}, \mathrm{YT}^{\mathrm{T}}$ is $\mathrm{YTaO}_{4}, \mathrm{YT}_{3}$ is $\mathrm{YTa}_{3} \mathrm{O}_{9}$ (Ref. 7).

phase, ${ }^{10}$ which is the product of the diffusionless phase transformation from the cubic phase to the tetragonal phase. The maximum $c / a$ is shifted to a lower temperature as $\mathrm{Y}_{2} \mathrm{O}_{3}$ composition increases. Based on these temperature- and compositiondependent tetragonality curves in the $\mathrm{ZrO}_{2}-\mathrm{Y}_{2} \mathrm{O}_{3}$ system, the anisotropic thermal expansion of the tetragonal phase will be discussed in this study. In the $\mathrm{ZrO}_{2}-\mathrm{YTaO}_{4}$ system, the tetragonal phase shows some interesting phenomena for its tetragonality and its $t \rightarrow m$ transformation temperature. Its anisotropic thermal expansions will be measured to compare with those in the $\mathrm{ZrO}_{2}-\mathrm{M}_{2} \mathrm{O}_{3}(\mathrm{M}=\mathrm{Y}, \mathrm{Yb}, \mathrm{In}, \mathrm{Sc})$ systems.

\section{Experimental Procedure}

Different compositions of tetragonal zirconia polycrystalline materials were produced by making fine powder, sintering, and heat-treating. Fine powder was prepared from precursor metal hydroxide using a coprecipitation method. Starting materials were $99.9 \%$ pure $\mathrm{M}_{2} \mathrm{O}_{3}(\mathrm{M}=\mathrm{Y}, \mathrm{Yb}, \mathrm{In}, \mathrm{Sc}), 99.9 \%$ pure $\mathrm{Ta}_{2} \mathrm{O}_{5}$, and $93.1 \%$ pure $\mathrm{ZrOCl}_{2}-8 \mathrm{H}_{2} \mathrm{O}$ (the remaining balance is $\left.\mathrm{H}_{2} \mathrm{O}\right) . \mathrm{M}_{2} \mathrm{O}_{3}$ and $\mathrm{Ta}_{2} \mathrm{O}_{5}$ were dissolved in hot acid $(\mathrm{HCl}$ or $\mathrm{HNO}_{3}$ ), and $\mathrm{ZrOCl}_{2}-8 \mathrm{H}_{2} \mathrm{O}$ was dissolved in distilled water. Two solutions, containing $\mathrm{M}^{+3}$ (or $\mathrm{Ta}^{+5}$ ) plus $\mathrm{Zr}^{+4}$ and $\mathrm{NH}_{4} \mathrm{OH}$ (10 wt $\%)$, were added simultaneously and mixed together to let a gellike metal hydroxide precipitate out by controlling the $\mathrm{pH}$ value above 9 . Precipitate was separated by filtration and was washed with water to eliminate chlorine ions. Then it was dried in an oven at $80^{\circ} \mathrm{C}$. Dried precipitates were calcined in an air oven for $2 \mathrm{~h}$ at $650^{\circ} \mathrm{C}$. Pellets were formed under a uniaxial pressure and were then isostatically pressed at $340 \mathrm{MPa}$. Subsequently, green pellets were sintered and heat-treated in air in a furnace with $\mathrm{MoSi}_{2}$ heating elements. 
For the lattice parameter measurement, samples in either a sintered powder or pellet form were used. Diffraction patterns of these samples were obtained using a high-temperature $\mathrm{X}$-ray diffractometer (Geigerflex, Rigaku, Tokyo, Japan). An $\mathrm{Al}_{2} \mathrm{O}_{3}$ plate was used as a sample holder and as an internal standard to calculate the lattice parameters. The reference diffraction peaks of $\mathrm{Al}_{2} \mathrm{O}_{3}$ were calibrated by pure Pt powder. The lattice constants of pure Pt metal at different temperatures were calculated from the thermal expansion data published by the National Bureau of Standards ${ }^{12}$ and from the Pt lattice constant at room temperature shown in JCPDS card number 4-802. Transformation temperatures between the tetragonal and the monoclinic phases were measured by a dilatometer unit. The operating temperature range was $25^{\circ}-1300^{\circ} \mathrm{C}$; heating and cooling rates were $10^{\circ} \mathrm{C} / \mathrm{min}$.

\section{Results}

\section{(I) Phase Continuity in the Composition Range 0-25 mol\% $\mathrm{YTaO}_{4}$}

A sample with $13.0 \mathrm{~mol} \% \mathrm{YTaO}_{4}$ contains both the tetragonal phase and monoclinic phase at room temperature. Its phase evolution at high temperatures is shown in Fig. 2. The characteristic monoclinic and tetragonal peaks $(11 \overline{1})_{m},(111)_{m},(111)_{t}$, $(004)_{t}$, and $(400)_{t}$ are used to identify these two phases. At $600^{\circ}$ and $900^{\circ} \mathrm{C}$, the tetragonal phase and the monoclinic phase coexist. At $1200^{\circ} \mathrm{C}$, only the tetragonal phase exists. This phase evolution indicates that the monoclinic phase at low temperatures is progressively transformed into the tetragonal phase at high temperatures. The same phase evolution is also found in the samples with composition less than $13.0 \mathrm{~mol}^{\circ} \mathrm{YTaO}_{4}$. The diffraction peaks in Fig. 2 shift to the low $2 \theta$ angle as the temperature increases, due to the larger lattice constants at high temperatures.

At $1200^{\circ} \mathrm{C}$, only the tetragonal phase is present in the composition range $0-25 \mathrm{~mol}_{\%} \mathrm{YTaO}_{4}$. Its lattice constants versus composition are shown in Fig. 3. Both lattice constants $c$ and $a$ are linearly dependent on composition. Figure 3 also shows the relationships between lattice constants and composition at $25^{\circ} \mathrm{C}$. Lattice constants for samples with composition less than $14.0 \mathrm{~mol}_{\%} \mathrm{YTaO}_{4}$ are obtained from data at high temperatures by using an extrapolation method, because the tetragonal phase is unstable at room temperature. A linear regression method indicates that pure tetragonal zirconia has lattice constants $a=$

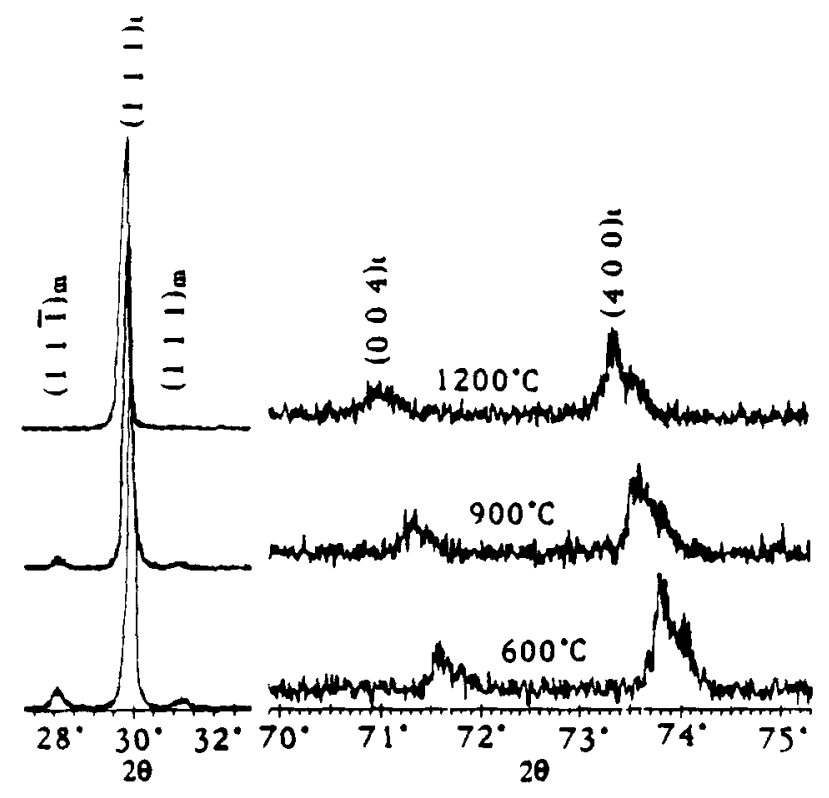

Fig. 2. X-ray diffraction patterns of a $13.0 \mathrm{~mol} \% \mathrm{YTaO}_{4}$ sample at three different temperatures.

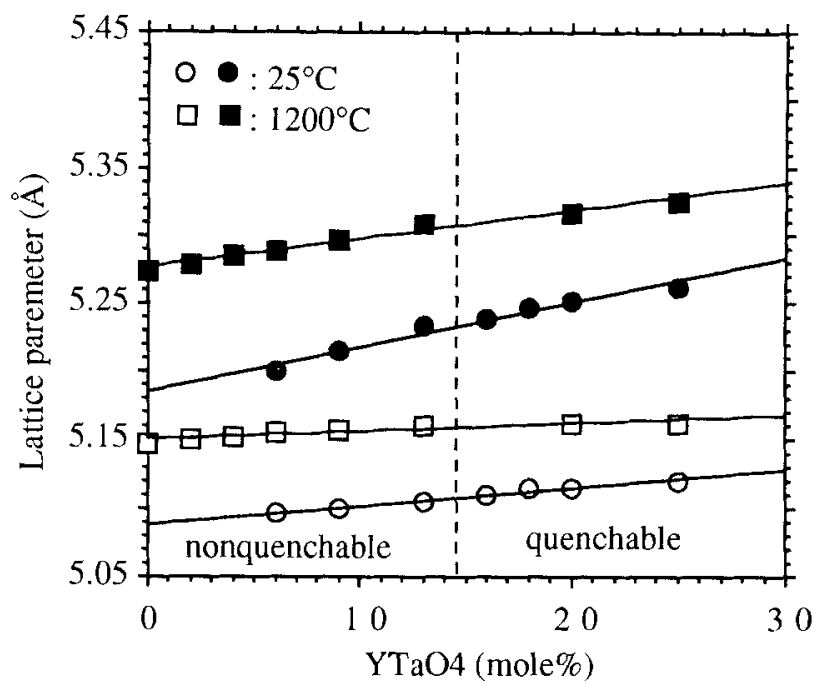

Fig. 3. Lattice parameters at $25^{\circ}$ and $1200^{\circ} \mathrm{C}$. Open symbols are for lattice constant $a$ and closed symbols are for lattice constant $c$.

$5.0887 \AA$ and $c=5.1849 \AA$ at $25^{\circ} \mathrm{C}$, which is consistent with previous data. ${ }^{3.4}$ Based on the above lattice constant continuities at $1200^{\circ} \mathrm{C}$ and at room temperature, it is suggested that only one tetragonal phase exists in the composition range $0-25$ $\mathrm{mol}_{\%} \mathrm{YTaO}_{4}$.

\section{(2) Tetragonality and Anisotropic Thermal Expansion Coefficients}

Tetragonality $(\mathrm{c} / \mathrm{a})$ in the $\mathrm{ZrO}_{2}-\mathrm{YTaO}_{4}$ and $\mathrm{ZrO}_{2}-\mathrm{M}_{2} \mathrm{O}_{3}$ systems at $1200^{\circ}$ and $25^{\circ} \mathrm{C}$ is presented in Fig. 4. As $\mathrm{YTaO}_{4}$ or $\mathrm{MO}_{1.5}$ composition increases, tetragonality increases in the $\mathrm{ZrO}_{2}-\mathrm{YTaO}_{4}$ system but it decreases in the $\mathrm{ZrO}_{2}-\mathrm{M}_{2} \mathrm{O}_{3}$ system. The slope of tetragonality versus composition, as indicated under each curve, is smaller at $1200^{\circ} \mathrm{C}$ than at $25^{\circ} \mathrm{C}$ in both cases. The $t \rightarrow m$ transformation temperature in these two cases, measured by the dilatometric method, is suppressed as $\mathrm{MO}_{1.5}$ or $\mathrm{YTaO}_{4}$ composition increases. It implies that the $t \rightarrow m$ transformation temperature in the $\mathrm{ZrO}_{2}-\mathrm{YTaO}_{4}$ system decreases with increasing tetragonality, but it is reversed in the $\mathrm{ZrO}_{2}-\mathrm{M}_{2} \mathrm{O}_{3}$.

Thermal expansion coefficients of the tetragonal phase versus its lattice constants are given in Fig. 5. The ordinate and the abscissa represent the average thermal expansion coefficients $\left(\alpha_{a}\right.$ or $\left.\alpha_{c}\right)$ in the two different crystallographic axes $(c$ or $a)$ at temperatures $900^{\circ}-1200^{\circ} \mathrm{C}$ and the lattice constants $(c$ or $a)$ at

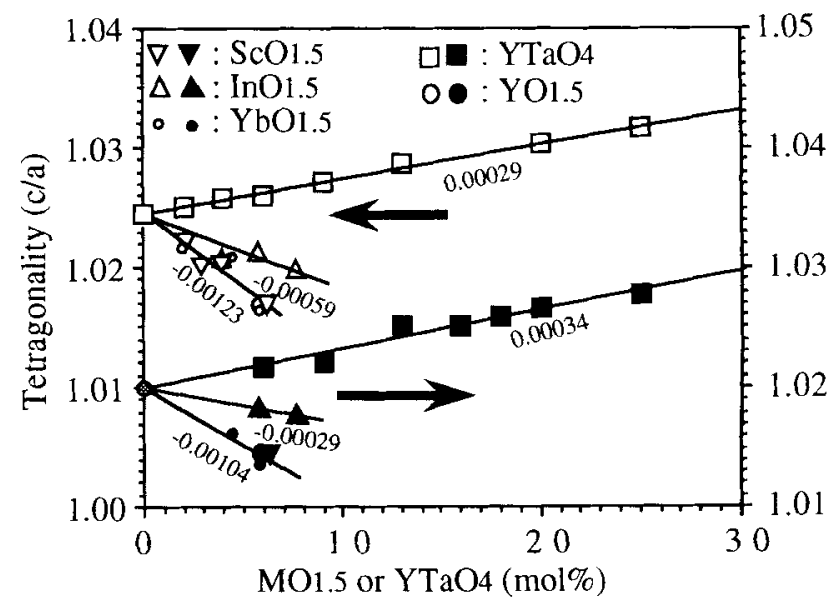

Fig. 4. Tetragonality in the $\mathrm{ZrO}_{2}-\mathrm{YTaO}_{4}$ and $\mathrm{ZrO}_{2}-\mathrm{MO}_{1.5}$. Open symbols are for temperature at $1200^{\circ} \mathrm{C}$ and full symbols are for temperature at $25^{\circ} \mathrm{C}$. Data under each curve is the slope of tetragonality versus composition. 


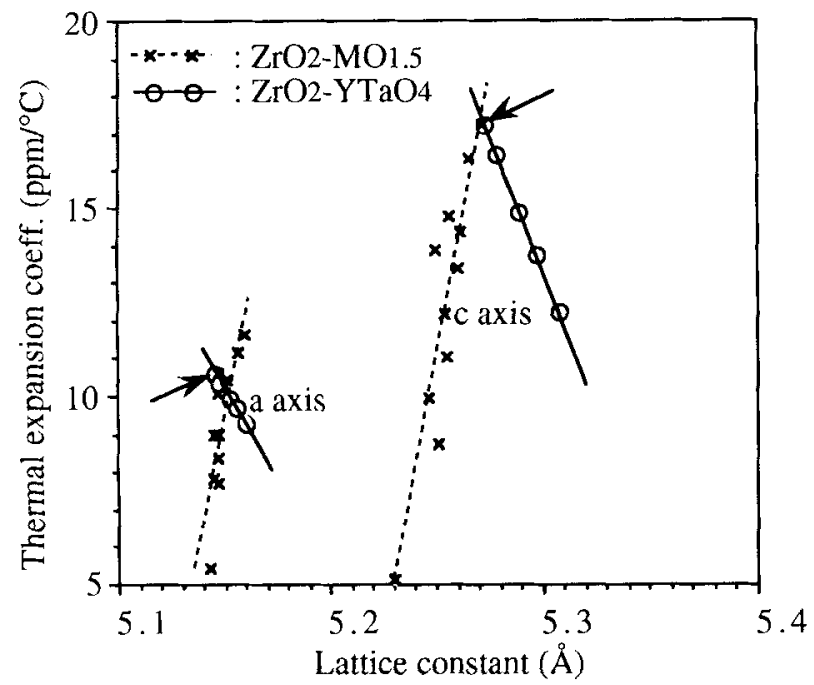

Fig. 5. Relationships between thermal expansion coefficient $(\alpha$ or $\alpha_{a}$ ) and lattice constant $\left(a\right.$ or $c$ ). Arrows indicate data for pure $\mathrm{ZrO}_{2}$.

$1200^{\circ} \mathrm{C}$, respectively. In $\mathrm{ZrO}_{2}-\mathrm{M}_{2} \mathrm{O}_{3}, \alpha_{a}$ and $\alpha_{c}$ increase with increasing lattice constants. It is reversed in the $\mathrm{ZrO}_{2}-\mathrm{YTaO}_{4}$ system. For the crystallographic a direction, pure zirconia has the highest thermal expansion coefficient in $\mathrm{ZrO}_{2}-\mathrm{YTaO}_{4}$ but not in $\mathrm{ZrO}_{2}-\mathrm{M}_{2} \mathrm{O}_{3}$. For the crystallographic $c$ direction, pure zirconia has the highest thermal expansion coefficient in both systems. The anisotropic thermal expansion coefficient ratio $\left(\alpha_{c} / \alpha_{a}\right)$ versus composition is shown in Fig. 6. $\alpha_{c} / \alpha_{u}$ decreases with increasing $\mathrm{MO}_{1.5}$ or $\mathrm{YTaO}_{4}$ composition. Schubert's data at $25^{\circ}-800^{\circ} \mathrm{C}$ for the $\mathrm{ZrO}_{2}-\mathrm{Y}_{2} \mathrm{O}_{3}$ system ${ }^{8}$ is close to $\mathrm{ZrO}_{2}-$ $\mathrm{Yb}_{2} \mathrm{O}_{3}$ in this study.

\section{Discussion}

\section{(I) Thermal Expansion in $\mathrm{ZrO}_{2}-\mathrm{Y}_{2} \mathrm{O}_{3}$}

Alderbert and Traverse ${ }^{9}$ and Sheu et al. ${ }^{10}$ have reported on the tetragonal phase ( $t$ or $t^{\prime}$ ) containing a temperature-dependent tetragonality, which first increases to a maximum and then decreases as temperature increases. Fundamentally, the $t$ and $t^{\prime}$ phases are the same tetragonal polymorph in the zirconia solid

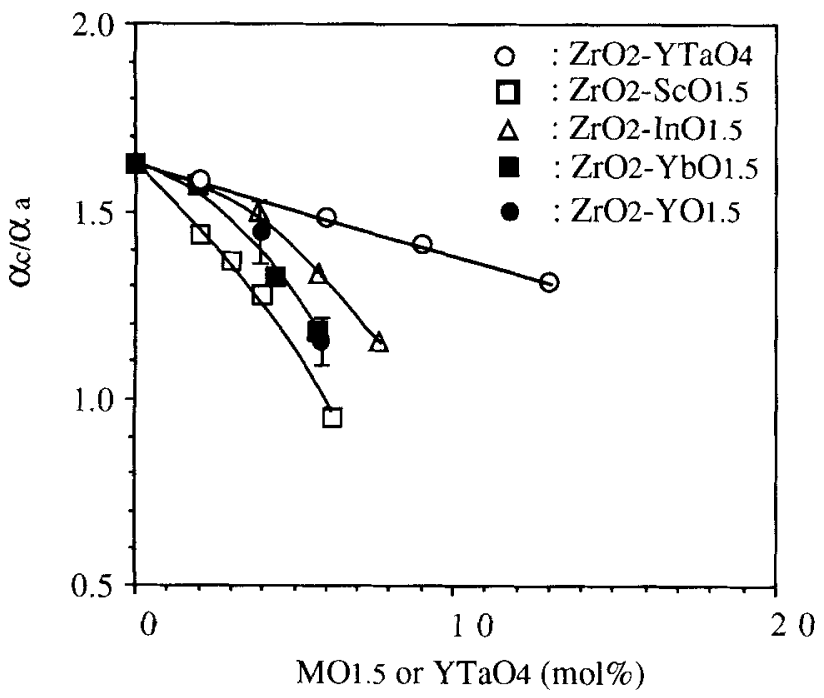

Fig. 6. Thermal expansion coefficient ratio $\left(\alpha_{t} / \alpha_{u}\right)$ versus composition in the temperature range $900^{\circ}-1200^{\circ} \mathrm{C}$. Data for $\mathrm{ZrO}_{2}-\mathrm{YO}_{1.5}$ are taken from Ref. 8 solutions. ${ }^{10}$ Based on these previous data, the thermal expansion behavior of the tetragonal phase in the $\mathrm{ZrO}_{2}-\mathrm{Y}_{2} \mathrm{O}_{3}$ system is discussed as follows.

There are two critical temperatures for each composition in this temperature-dependent tetragonality curve. One is at $T_{\mathrm{c}}$, the transformation temperature of the cubic phase $(c)$ to the tetragonal phase ( $t$ or $t^{\prime}$ ). At $T_{\mathrm{c}}$, the tetragonal phase shows a tetragonality anomaly, which decreases from $c / a \neq 1$ to $c / a=$ 1. ${ }^{911}$ This $T_{\text {c }}$ temperature has been measured for the pure zirconia and the sample with $13.5 \mathrm{~mol} \% \mathrm{YO}_{1.5}$. The other critical temperature is at $T_{\mathrm{a}}$ which has a maximum tetragonality $(c / a)_{\max }$. At $T_{\mathrm{a}}$, this temperature-dependent tetragonality $(c / a=f(T))$ has the following relationships: $f^{\prime}(T)=0$, and $f^{\prime \prime}(T)<0$. Alternatively, $f^{\prime}(T)=0$ can be expressed as $\partial f(T) / \partial T=\partial(c / a) / \partial T=0$. From $\partial(c / a) / \partial T=0$, we obtain $(\partial c / \partial T) \times a-(\partial a / \partial T) \times c=0$. Subsequently, $(\partial c / \partial T) / c=$ $(\partial a / \partial T) / a$, i.e., $\alpha_{c}=\alpha_{a}$, is obtained at $T_{\mathrm{a}}$. This derivation indicates that the tetragonal phase has the same thermal expansion coefficients in the crystallographic $a$ and $c$ directions when its tetragonality reaches a peak maximum, $(c / a)_{\max }$.

Based on Alderbert's and Sheu's experimental data, ${ }^{9.10}$ a $\mathrm{ZrO}_{2}-\mathrm{Y}_{2} \mathrm{O}_{3}$ phase diagram containing $(c / a)_{\max }, \alpha_{c}=\alpha_{a}, c / a=$ $1, T_{u}$, and $T_{c}$ is shown in Fig. 7. Several published phase boundaries of the tetragonal and cubic phases ${ }^{13-16}$ are also included. Symbols " $*$ " are used to represent $T_{\mathrm{c}}$ temperatures. There are three data points for $c / a=1$, at $25^{\circ}, 600^{\circ}$, and $1200^{\circ} \mathrm{C}$, respectively. ${ }^{10}$ This $c / a=1$ curve at high temperatures is close to one of the cubic phase boundaries. ${ }^{16}$ Compared with the $c / a=1$ curve, the $\alpha_{c}=\alpha_{a}$ or $(c / a)_{\max }$ curve is very far from the cubic phase boundary. This locus of $\alpha_{c}=\alpha_{a}$ represents only a maximum tetragonality for the tetragonal phase, but not the phase boundary for the cubic phase. This is why the data point of $\alpha_{c}=\alpha_{a}$ does not match the cubic phase boundary in Schubert's paper."

\section{(2) Thermal Expansion in $\mathrm{ZrO}_{2}-\mathrm{M}_{2} \mathrm{O}_{3}$ and $\mathrm{ZrO}_{2}-\mathrm{YTaO}_{4}$}

By introducing the lattice constants from a linear regression method and the lattice constants of pure zirconia ${ }^{3}$ at $25^{\circ} \mathrm{C}$ in $\mathrm{ZrO}_{2}-\mathrm{M}_{2} \mathrm{O}_{3}$ and $\mathrm{ZrO}_{2}-\mathrm{YTaO}_{4}$, the average thermal expansion coefficients $\left(\alpha_{c}\right.$ or $\alpha_{4}$ ) of the tetragonal phase at $25^{\circ}-1200^{\circ} \mathrm{C}$ versus its composition are shown in Fig. 8. For $\alpha_{c}$, it decreases with increasing $\mathrm{MO}_{1.5}$ or $\mathrm{YTaO}_{4}$ composition. For $\alpha_{a}$, it increases with increasing $\mathrm{MO}_{1.5}$ composition (except for $\mathrm{ScO}_{1.5}$ ) but decreases with increasing $\mathrm{YTaO}_{4}$. Although thermal expansion coefficients $\left(\alpha\right.$, and $\left.\alpha_{a}\right)$ versus composition behave differently, the thermal expansion coefficient difference $\Delta \alpha\left(=\alpha_{c}-\right.$

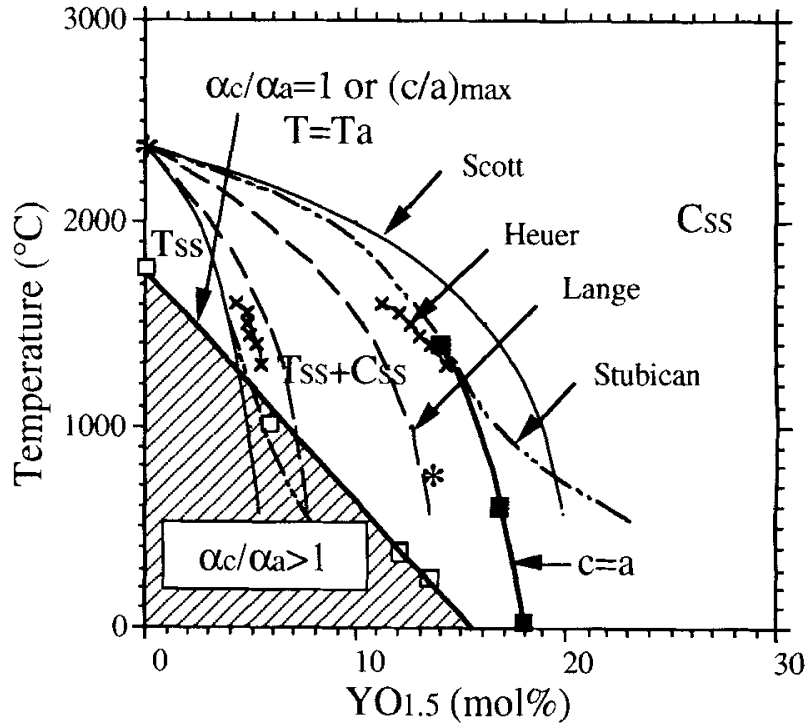

Fig. 7. Diagram showing the relationships among $\alpha_{c} / \alpha_{n}=1$, $(c / a)_{\max }$, and $c=a$. Tss is $t-\mathrm{ZrO}_{2}$ solid solution, Css is $c-\mathrm{ZrO}_{2}$, solid solution, $T$ is temperature. Symbols "*" are for temperatures $T_{\mathrm{c}}$. 


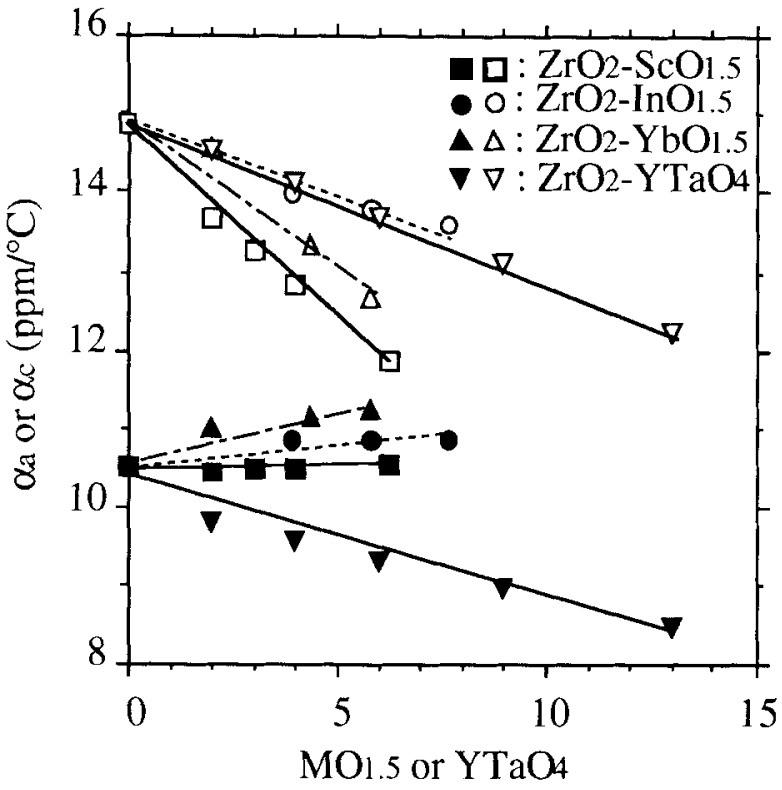

Fig. 8. Average thermal expansion coefficient at $25^{\circ}-1200^{\circ} \mathrm{C}$. Open symbols are for $\alpha_{t}$ and closed symbols are for $\alpha_{t i}$.

$\alpha_{a}$ ) versus composition in these two systems shows the same trend, that is, it decreases with $\mathrm{MO}_{1.5}$ or $\mathrm{YTaO}_{4}$ composition. Compared with $\mathrm{ZrO}_{2}-\mathrm{Sc}_{2} \mathrm{O}_{3}$ and $\mathrm{ZrO}-\mathrm{Yb}_{2} \mathrm{O}_{3}, \mathrm{ZrO}_{2}-\mathrm{In}_{2} \mathrm{O}_{3}$ and $\mathrm{ZrO}_{2}-\mathrm{YTaO}_{4}$ show a smaller change of $\Delta \alpha$ versus composition.

The relationships among the thermal expansion coefficient, the lattice constant, and composition in $\mathrm{ZrO}_{2}-\mathrm{YTaO}_{4}$ and $\mathrm{ZrO}_{2}-$ $\mathrm{M}_{2} \mathrm{O}_{3}$ are discussed as follows. For the thermal expansion coefficient $\left(\alpha_{a}\right.$ or $\alpha_{c}$ ) versus lattice constant ( $a$ or $c$ ) shown in Fig. 5, $\left(\partial \alpha_{\alpha} / \partial c\right)>0$ and $\left(\partial \alpha_{a} / \partial a\right)>0$ in $\mathrm{ZrO}_{2}-\mathrm{M}_{2} \mathrm{O}_{3}$, and $\left(\partial \alpha_{c} / \partial c\right)<0$ and $\left(\partial \alpha_{a} / \partial a\right)<0$ in $\mathrm{ZrO}_{2}-\mathrm{YTaO}_{4}$. For the lattice constant $(c$ or a) versus composition (X) shown in Refs. 3 and 10 and in Fig. $3, \partial c / \partial \mathrm{X}<0$ (except for $\mathrm{M}=\mathrm{Nd}$, when $\partial c / \partial \mathrm{X}>0)^{3}$ and $\partial a / \partial \mathrm{X}>0$ (except for $\mathrm{M}=\mathrm{Sc}$, when $\partial a / \partial \mathrm{X} \leq 0)^{10}$ in $\mathrm{ZrO}_{2}$ $\mathrm{M}_{2} \mathrm{O}_{3}$, and $\partial c / \partial \mathrm{X}>0$ and $\partial a / \partial \mathrm{X}>0$ in $\mathrm{ZrO}_{2}-\mathrm{YTaO}_{4}$. From the above two relationships, it can be inferred that for the thermal expansion coefficient $\left(\alpha_{a}\right.$ or $\left.\alpha_{c}\right)$ versus composition (X), $\left(\partial \alpha_{1} / \partial \mathrm{X}\right)<0$ and $\left(\partial \alpha_{1 /} / \partial \mathrm{X}\right)>0$ in $\mathrm{ZrO}_{2}-\mathrm{M}_{2} \mathrm{O}_{3}$ (except for $\mathrm{M}=$ $\mathrm{Nd}, \mathrm{Sc})$, and $\left(\partial \alpha_{i} / \partial \mathrm{X}\right)<0$ and $\left(\partial \alpha_{4} / \partial \mathrm{X}\right)<0$ in $\mathrm{ZrO}_{2}-\mathrm{YTaO}_{4}$. A schematic diagram to represent these relationships is given in Fig. 9. There exists $c=a$ in $\mathrm{ZrO}_{2}-\mathrm{M}_{2} \mathrm{O}_{3}$, but not in $\mathrm{ZrO}_{2}-$ $\mathrm{YTaO}_{4} \cdot \alpha_{c} / \alpha_{a}=1$ is present in both systems. By extrapolating data to $\alpha_{i} / \alpha_{i s}=1$ in Fig. 6 , the locus of $\alpha_{c}=\alpha_{a}$ at $900^{\circ}-$ $1200^{\circ} \mathrm{C}$ in $\mathrm{ZrO}_{2}-\mathrm{M}_{2} \mathrm{O}_{3}$ is approximately at $6 \mathrm{~mol} \% \mathrm{ScO}_{1.5}, 7$ mol\% $\mathrm{YbO}_{1.5}$, and $9 \mathrm{~mol} \% \mathrm{InO}_{1.5}$, respectively. This $\alpha_{s} / \alpha_{a}=1$ is far from the cubic phase boundary in $\mathrm{ZrO}_{2}-\mathrm{M}_{2} \mathrm{O}_{3}$. In $\mathrm{ZrO}_{2}-$ $\mathrm{YTaO}_{4}$, the locus of $\alpha_{c^{\prime}}=\alpha_{a}$ is approximately at $25 \mathrm{~mol} \%$ $\mathrm{YTaO}_{4}$ at $900^{\circ}-1200^{\circ} \mathrm{C}$, even though the cubic phase does not exist in this system. This locus of $\alpha_{c}=\alpha_{a}$ is close to the tetragonal phase boundary at $900^{\circ}-1200^{\circ} \mathrm{C}$ in $\mathrm{ZrO}_{2}-\mathrm{YTaO}_{4}$.

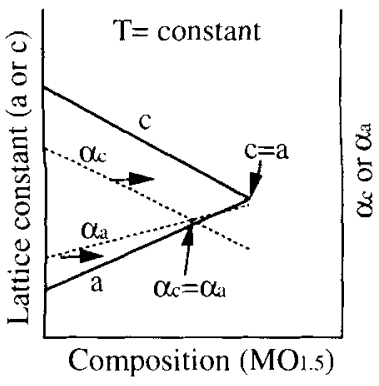

(a)

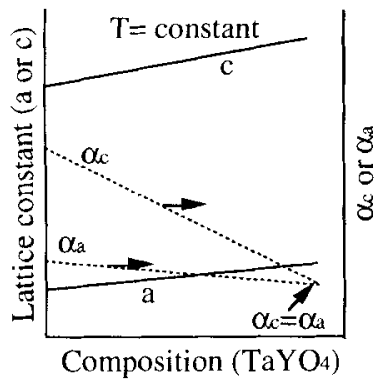

(b)
Fig. 9. Relationships among lattice constant, thermal expansion, and composition in the (a) $\mathrm{ZrO}_{2}-\mathrm{M}_{2} \mathrm{O}_{3}$ and (b) $\mathrm{ZrO}-\mathrm{YTaO}_{4}$ systems.
Tetragonality is related to the magnitude of oxygen sheartype shifting in [001] $\{110\} .^{17}$ This oxygen shifting produces two different $\mathrm{Zr}-\mathrm{O}$ bonds, four short ones and four long ones, for each $\mathrm{Zr}$ atom. In pure zirconia, Alderbert and Traverse ${ }^{y}$ have found that the long $\mathrm{Zr}-\mathrm{O}$ bond has a higher thermal expansion coefficient up to a certain temperature, and then it is reversed because this long $\mathrm{Zr}-\mathrm{O}$ bond becomes unstable. This phenomenon causes the presence of $(c / a)_{\max }$ or $\alpha_{c}=\alpha_{a}$ in the curve of tetragonality versus temperature. The presence of $\alpha_{c}=\alpha_{a}$ in the $\mathrm{ZrO}_{2}-\mathrm{M}_{2} \mathrm{O}_{3}$ and $\mathrm{ZrO}_{2}-\mathrm{YTaO}_{4}$ systems suggests that the bond length change due to the effect of temperature in these two systems behaves like that in pure zirconia. However, why the thermal expansion coefficients of the tetragonal phase decrease with increasing lattice constants in the $\mathrm{ZrO}_{2}-\mathrm{YTaO}_{4}$ system is not fully understood.

\section{(3) Thermal Stresses}

In the tetragonal zirconia polycrystals, partially stabilized zirconia, and zirconia-toughened ceramic materials, ${ }^{11.18-29}$ the thermal stresses from different kinds of mismatch (volume, expansion, etc.) have been shown to affect the transformability of tetragonal grains (or particles) in these materials. In the classical nucleation theory, the thermal stresses have accounted for the stress-induced transformation from the tetragonal $(t)$ to the monoclinic $(m)$ phase. ${ }^{21.22}$ The anisotropic thermal expansion causes thermal stresses among differently orientated grains in the tetragonal zirconia polycrystals. Some grains are locally subjected to high thermal stresses. With increasing grain size, these thermal stresses are further increased up to the critical stresses to trigger the $t \rightarrow m$ transformation. In $\mathrm{ZrO}_{2}-\mathrm{M}_{2} \mathrm{O}_{3}$ and $\mathrm{ZrO}_{2}-\mathrm{YTaO}_{4}$, the anisotropic thermal expansion coefficient difference $\left(\Delta \alpha=\alpha_{c}-\alpha_{i}\right)$ in the two crystallographic axes of the tetragonal phase decreases with $\mathrm{MO}_{1.5}$ or $\mathrm{YTaO}_{4}$ content. Lower thermal stresses are expected to exist in the tetragonal polycrystals with a higher $\mathrm{MO}_{15}$ or $\mathrm{YTaO}_{4}$ content. As a result, the $t \rightarrow$ $m$ transformation is more difficult to trigger by these thermal stresses.

Anisotropic thermal expansion is also temperature-dependent. A transition temperature $T_{\mathrm{a}}$ has been shown in Fig. 7 . When $T<T_{a}$, then $\alpha_{c}>\alpha_{a}$; when $\mathrm{T}>T_{a}$, then $\alpha_{c}<\alpha_{a}$. This reverse anisotropic thermal expansion behavior changes the magnitude of thermal stresses for the tetragonal zirconia polycrystals. For example, with the same grain size, tetragonal polycrystalline material being heat-treated above $T_{\mathrm{a}}$ and then quenched to the ambient temperature has lower thermal stresses than the sample being heat-treated at $T_{x}$. This is because the thermal stresses arising from cooling from $T_{\mathrm{a}}$ to the ambient temperature are relaxed by the reverse type of stresses arising from the heat-treating temperature $T$ to $T_{i}$. Because of this characteristic curve of $\alpha_{c} / \alpha_{a}$ versus temperature, a post-heat treatment just below temperature $T_{\mathrm{a}}$ for a long period of time is suggested to improve the transformability of the tetragonal polycrystals.

\section{Conclusions}

(1) From the lattice parameter measurements at high temperatures, a single tetragonal phase is determined to exist from 0-25 mol\% $\mathrm{YTaO}_{4}$ in the $\mathrm{ZrO}_{2}-\mathrm{YTaO}_{4}$ system. Its tetragonality $(c / a)$ is larger than that in pure zirconia and increases with increasing $\mathrm{YTaO}_{4}$ composition. However, with composition higher than $15 \mathrm{~mol}_{\%} \mathrm{YTaO}_{4}$, this tetragonal phase is stable at room temperature.

(2) Anisotropic thermal expansion coefficients of the tetragonal phase are measured at $25^{\circ}-1200^{\circ} \mathrm{C}$ in $\mathrm{ZrO}_{2}-\mathrm{M}_{2} \mathrm{O}_{3}$ and $\mathrm{ZrO}_{2}-\mathrm{YTaO}_{4}$. Thermal expansion coefficients, $\alpha_{c}$ and $\alpha_{a}$, vary with $\mathrm{M}_{2} \mathrm{O}_{3}$ or $\mathrm{YTaO}_{4}$ composition. However, the ratio and the difference of these two thermal expansion coefficients, $\alpha_{i} / \alpha_{i i}$ and $\alpha_{i}-\alpha_{a}$, decrease with increasing $\mathrm{M}_{2} \mathrm{O}_{3}$ or $\mathrm{YTaO}_{4}$ composition, respectively.

(3) From the relationships among thermal expansion, temperature, and composition, the locus of $\alpha_{c}=\alpha_{a}$ exists in both 
$\mathrm{ZrO}_{2}-\mathrm{M}_{2} \mathrm{O}_{3}$ and $\mathrm{ZrO}_{2}-\mathrm{YTaO}_{4}$. This locus of $\alpha_{c}=\alpha_{a}$ indicates a maximum tetragonality $\left((c / a)_{\max }\right)$ for the tetragonal phase, but not the phase boundary for the cubic phase.

\section{References}

'M. Marezio, "Refinement of the Crystal Structure of In, $\mathrm{O}_{3}$ at Two Wavelengths," Acta Crystallogr., 20, 723-28 (1966)

2D. K. Hotnke, "Ionic Conductivity of $\mathrm{Zr}_{1-2} \ln _{2,} \mathrm{O}_{2}$,"J. Phys. Chem. Solids, 41, 777-84 (1980).

M. Yoshimura, "Phase Stability of Zirconia," Am. Ceram. Soc. Bull., 67 [12] 1950-55 (1988)

J. Lefevre, "Fluorite-Type Structural Phase Modification in Systems Having a Zirconium or Hafnium Oxide Base," Ann. Chim. (Paris) (in Fr.) 8 [1-2] 11749 (1963); or translated by A. L. Monka, Report No. ORNL-TR-201.

${ }^{S} \mathrm{R}$. R. Lee, and A. H. Heuer, "Morphology of Tetragonal $\mathrm{ZrO}_{2}$ in a Ternary (Mg, Y)-PSZ,"J. Am. Ceram. Soc., 70 [4] 208-13 (1987).

"D. J. Kim "Effect of $\mathrm{Ta}_{2} \mathrm{O}_{5}, \mathrm{Nb}_{2} \mathrm{O}_{4}$ and $\mathrm{HfO}_{2}$ Alloying on the Transformability of $\mathrm{Y}_{2} \mathrm{O}_{3}$-Stabilized Tetragonal $\mathrm{ZrO}_{2}, " J$. Am. Ceram. Soc., 73 [1] 115-20(1990)

${ }^{7}$ D. J. Kim, and T. Y. Tien, "Phase Stability and Physical Properties of Cubic and Tetragonal $\mathrm{ZrO}$, in the System $\mathrm{ZrO},-\mathrm{Y}_{2} \mathrm{O}_{2}-\mathrm{Ta}_{2} \mathrm{O}_{5}, " J$. Am. Ceram. Soc., 74 [12] 3061-65 (1991).

${ }^{8} \mathrm{H}$. Schubert, "Anisotropic Thermal Expansion Coefficients of $\mathrm{Y}_{2} \mathrm{O}_{3}$-Stabilized Tetragonal Zirconia," J. Am. Ceram. Soc., 69 [3]270-7] (1986).

"P. Alderbert, and J. P. Traverse, "Structure and Ionic Mobility of Zirconia at High Temperature," J. Am. Ceram. Soc., 68 [1] 34-40 (1985).

iT. S. Sheu, T. Y. Tien, and I-W. Chen, "Cubic-to-Tetragonal $\left(t^{\prime}\right)$ Transformation in Zirconia-Containing Systems," J. Am. Ceram. Soc., 75 [5] 1108-16 (1992).

"A. H. Heuer, and M. Ruhle. "On the Nucleation of the Martensitic Transformation in Zirconia ( $\mathrm{ZrO}_{2}$ )," Acta Metall, 33 [12] 2101-12 (1985).
${ }^{12}$ T. A, Hahn, and R. K. Kirby, "Thermal Expansion of Platinum from 293 to $1900^{\circ} \mathrm{K}$." Institute of Materials Research, National Bureau of Standards, Washington, DC, 1971.

"H. G. Scott, "Phase Relations in the Zirconia-Yttria System," J. Mater. Sci., 10 [9] 1527-35 (1975).

${ }^{14} \mathrm{~A}$. H. Heuer, and $\mathrm{M}$. Ruhle, "Phase Transformations in $\mathrm{ZrO}_{2}$-Containing Ceramics: I, The Instability of $c-\mathrm{ZrO}_{2}$ and the Resulting Diffusion-Controlled Reactions": pp. 1-13 in Advances in Ceramics, Vol. 12, Science and Technology of Zirconia II. Edited by N. Claussen, M. Ruhle, and A. H. Heuer. American Ceramic Society, Columbus, $\mathrm{OH}, 1984$.

"F. F. Lange, "Transformation Toughening: Thermodynamic Approach to the Phase Retention and Toughening"; pp. 255-74 in Fracture Mechanics of Ceramics, Vol. 6. Edited by R. C. Bradt, A. G. Evans, D. P. H. Hasselmann, and F. F. Lange. Plenum Press, New York, 1983.

inV. S. Stubican. G. S. Corman, J. R. Hellmann, and G. Senft, "Phase Relationship in Some ZrO $\mathrm{Z}_{2}$ Systems"; see Ref. 12, pp. 96-106.

${ }^{17} \mathrm{G}$. Teufer, "The Crystal Structure of Tetragonal $\mathrm{ZrO}_{2}$, " Acta Crystallogr. $15,1187(1962)$

${ }^{11 R}$ R. C. Garvie and M. V. Swain, "Thermodynamics of the Tetragonal-toMonoclinic Phase Transformation in Constrained Zirconia Microcrystals." J. Mater. Sci., 20, I193-200 (1985).

${ }^{14} \mathrm{~S}$. Schmauder, W. Mader, and M. Ruhle, "Calculations of Strain Distributions in and around $\mathrm{ZrO}_{2}$ Inclusions"; see Ref. 14, pp. 251-55.

${ }^{37}$ A. H. Heuer, M. Ruhle, and D. B. Marshall, "On the Thermoelastic Martensitic Transformation in Tetragonal Zirconia," J. Am. Ceram. Soc., 73 [4] $1084-93(1990)$.

"I-W. Chen and Y. H. Chiao, "Martensitic Nucleation in $\mathrm{ZrO}_{2}$," Acta Metall., $31[10] 1627-38$ (1983).

"Y. H. Chiao, "Nucleation and Growth Processes of Martensitic Transformation in $\mathrm{ZrO}_{2}$ Particles"; $\mathrm{Ph} . \mathrm{D}$. Thesis. Massachusetts Institute of Technology, Cambridge, MA, Sept. 1986. 